\title{
REVIEW
}

\section{A Review of Uric Acid, Crystal Deposition Disease, and Gout}

Fernando Perez-Ruiz $\cdot$ Nicola Dalbeth $\cdot$ Tomas Bardin

To view enhanced content go to www.advancesintherapy.com

Received: November 13, 2014 / Published online: December 23, 2014

(c) The Author(s) 2014. This article is published with open access at Springerlink.com

\section{ABSTRACT}

There has been increased interest in gout in both academic and clinical practice settings. Several reasons may explain this. The prevalence of both hyperuricemia and gout has risen in the last decades in developed countries and therefore the burden of gout has increased. The association of hyperuricemia and gout with cardiovascular outcomes and the opportunity of further benefits of intervention on hyperuricemia have been recently highlighted in the literature. Imaging techniques have proven to be useful for

Electronic supplementary material The online version of this article (doi:10.1007/s12325-014-0175-z) contains supplementary material, which is available to authorized users.

F. Perez-Ruiz ( $\square)$

Servicio de Reumatologia, Hospital Universitario Cruces, and BioCruces Health Research Institute, 48903 Baracaldo, Vizcaya, Spain

e-mail: fernando.perezruiz@osakidetza.net

N. Dalbeth

University of Auckland, Auckland, New Zealand

T. Bardin

Hopital Lariboisiere, Universite Paris VII Diderot, Paris, France detection of urate deposition, even prior to the first clinical symptoms, enabling the evaluation of the extent of deposition and providing objective measurement of crystal depletion during urate-lowering treatment. Treating to target is increasingly used as the approach to treatment of diverse diseases. Therefore, different targets have been recommended for different stages of the burden of disease and for different stages of treatment. The final strategic target, to which any effort should be taken into consideration, is to completely dissolve urate crystals in tissues and therefore avoid further symptoms and structural damage of involved musculoskeletal structures. In summary, evidence suggest that an early approach to the treatment of gout and associated comorbidities is advisable, that new imaging techniques may help to evaluate both the burden of deposition and response to uratelowering treatment in selected patients, and finally that the final strategic objective of healthcare for patients with gout is to completely resolve urate crystal deposits.

Keywords: Crystal deposition disease; Gout; Hyperuricemia; Uric acid 


\section{URIC ACID, GOUT, AND CARDIOVASCULAR DISEASES: THE SILENT EPIDEMIC}

An increase in the prevalence of both hyperuricemia and gout has been shown during the previous decades in developed countries. The association of hyperuricemia, but especially of gout, with cardiovascular outcomes and the opportunity of further benefits of early intervention have been recently highlighted. The fact that crystal deposition and subclinical inflammation precede the clinical onset of gout may deliver a new approach to the treatment of hyperuricemia and gout.

Gout is due to the nucleation and growth of monosodium urate (MSU) crystals in tissues in and around the joints, following long-standing hyperuricemia, that is, serum urate (sUA) above the saturation threshold.

The sequence of events that occur in the hyperuricemic patients has been recently suggested to comprise four stages (Fig. 1) [1]. Hyperuricemia promotes MSU crystal deposits that are first asymptomatic but can be detected by imaging techniques such as ultrasound and dual-energy computed tomography (DECT). Once formed, MSU crystals can cause acute, self-limited, inflammatory flares, probably triggered by crystal shedding from the cartilage surface into the joint space, where they can interact with resident cells. If hyperuricemia persists, MSU crystal deposits further induce chronic inflammatory responses that may lead to damage of the joint structures, the so-called chronic gouty arthritis or chronic gout, which is usually associated with the presence of subcutaneous MSU deposits or tophi. Finally, as deposits grow and comorbidities increase, gout becomes even more severe and difficult to treat becoming what is known as "refractory gout".

Therefore, gout must be viewed as a chronic, progressive disease if sUA levels are not appropriately lowered. MSU crystal deposits

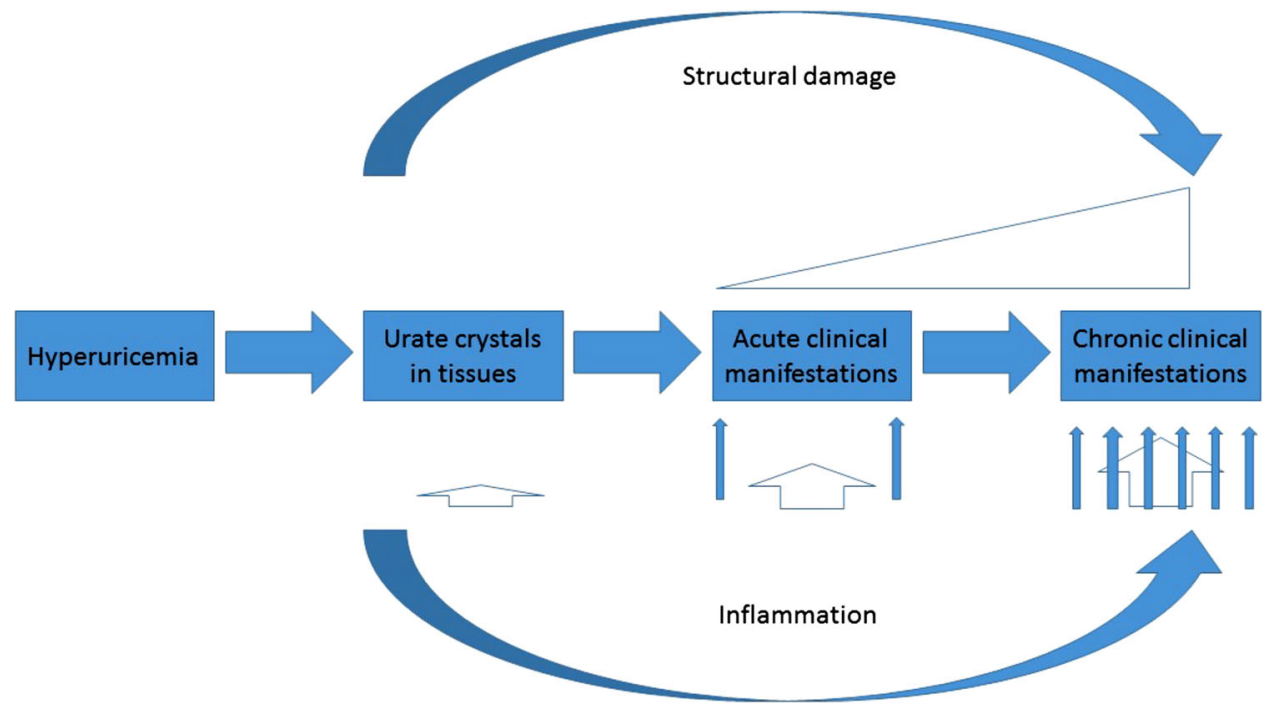

Fig. 1 Diagram showing the path from hyperuricemia to structural joint damage. Long-standing hyperuricemia may lead to monosodium urate deposits, with no symptoms and sometimes associated to subclinical inflammation; intermittent acute inflammation is caused by crystal shedding into the joint space, inflammation persisting between flares, bone erosions may appear; untreated or improperly treated deposition will lead to further persistent inflammation, increase in the number of flares and structural joint damage with permanent joint space narrowing 
should be considered the prime and most important target for the management of gout. By lowering sUA levels at least below $6 \mathrm{mg} / \mathrm{dl}$ $(360 \mu \mathrm{ml} / \mathrm{L})$, dissolution of the pathogenic MSU crystals is achieved and disappearance of clinical features of gout can be obtained [2, 3]. This chronic disease should be taken seriously, as it can cause severe damage to joint structures and has also been associated with poor kidney and cardiovascular outcomes [4].

Recent data have shown that hyperuricemia and gout are increasing worldwide [5]. Such an epidemic has deleterious consequences not only on joint function, health resources utilization, and quality of life, but may also increase cardiovascular mortality. Gouty patients die mainly from cardiovascular events [6], and several studies have shown that gout is indeed an independent cardiovascular risk factor [7-9], even more when gout is severe [6]. This independent effect of gout on cardiovascular mortality adds on frequently associated comorbidities; although it may reflect to some extent frequent nonsteroidal anti-inflammatory drugs (NSAIDs) intake during flares, inflammation and hyperuricemia are considered to be the main factors [4].

Asymptomatic hyperuricemia has long been known to be associated with cardiovascular morbidity and mortality, but the traditional view has been that this was due to the association of well-known cardiovascular risk factors, as adjustment for associated traditional risk factors led to the disappearance of the association between hyperuricemia and cardiovascular events in the Framingham heart study [10]. Asymptomatic hyperuricemia has long been considered as a mere indicator of associated cardiovascular comorbidities, the frequency of which correlates with the level of hyperuricemia [11]. Comorbidities and not hyperuricemia per se were believed to explain the increased death rate. This view has been very much challenged during the last decade, as recent studies have shown an independent association of hyperuricemia with cardiovascular events, although this association appeared to be weaker than with gout [4]. Moreover, data have accumulated which suggests that hyperuricemia plays a role in the occurrence of hypertension, heart disease, type 2 diabetes and renal impairment [4], which would make traditional statistical adjustments for confounding variables inaccurate. For example, if hyperuricemia causes hypertension, adjustment for hypertension to determine whether hyperuricemia independently associates with cardiovascular outcomes makes little sense.

Indeed, hyperuricemia has been shown to precede hypertension in many prospectively followed human cohorts [12]. Several animal studies, using various ways to induce hyperuricemia, demonstrated that hyperuricemia induced hypertension in rats and blood pressure could be corrected by various urate-lowering drugs if these were given early [13-15]. Similarly, in hyperuricemic adolescents with incipient hypertension or prehypertension, uratelowering medications (ULMs) normalized blood pressure [16, 17]. Hyperuricemia is frequently associates with type 2 diabetes and the metabolic syndrome [11], and prospective human studies have shown that hyperuricemia again preceded the appearance of diabetes [4]. In rats fed with a fructose-enriched diet, hyperuricemia and hyperinsulinism developed and were corrected by urate-lowering interventions [14]. The detrimental effect of hyperuricemia on the kidney is suggested by animal studies [13]. Human studies have shown that hyperuricemia predicted the appearance of chronic kidney disease (CKD) [18] and was a 
factor of poor prognosis in many renal diseases [4]. In addition, small randomized controlled trials have suggested that sUA lowering might improve kidney prognosis in CKD patients [4].

The beneficial effect of sUA lowering on cardiovascular mortality has not been studied in randomized controlled trials (RCTs). However, several retrospective studies have concluded that allopurinol intake was associated with a decrease in myocardial infarction and mortality $[4,19]$, especially when allopurinol was given at a dose allowing reduction of sUA below $6 \mathrm{mg} / \mathrm{dL}$ [20].

Therefore, despite some ongoing controversies, the case is strong for considering hyperuricemia as an independent cardiovascular risk factor. However, because of the lack of large RCTs showing that urate lowering improves cardiovascular mortality, no drug has been thus far approved, apart from in Japan, for the management of asymptomatic hyperuricemia [4].

\section{TAKING A NEW LOOK AT GOUT: THE ROLE OF IMAGING IN THE CLINICAL MANAGEMENT OF GOUT}

Imaging techniques have nicely shown the pathophysiological connection between hyperuricemia, urate crystal deposition in tissues, and gout, even prior to the first clinical symptom. They have been shown to be sensitive to detect the extent of deposition, but also sensitive enough to change to provide objective measurement of crystal depletion during urate-lowering treatment.

Advanced imaging modalities have provided important new insights into the mechanisms of disease and treatment approaches in gout. As described above, the central feature of gout is deposition of MSU crystals, and the clinical manifestations of gout occur due to the individual's response to these crystals. The most widely studied imaging methods that allow analysis of MSU crystal deposition are ultrasonography and DECT. On ultrasonography, MSU crystals overlying the articular cartilage can be detected as the 'double contour' sign [21, 22]. Tophi, collections of MSU crystals surrounded by an organized cellular rim and fibrovascular zone [23], can also be detected by ultrasonography [21]. Dual-source DECT, a dual-energy computerized tomography (CT) technique that includes two X-ray sources and two detectors, evaluates the different attenuations of the scanned object to analyze if the chemical composition of materials includes urate crystals. In gout, urate can be color-coded for visualization and measured using automated volume measurement software [24] (Fig. 2).

A key insight into gout pathogenesis that has arisen from ultrasonography studies is the observation that MSU crystals are present in some people with hyperuricemia, but without clinical symptoms of gout. It has long been recognized that although hyperuricemia is virtually always present in people with gout,

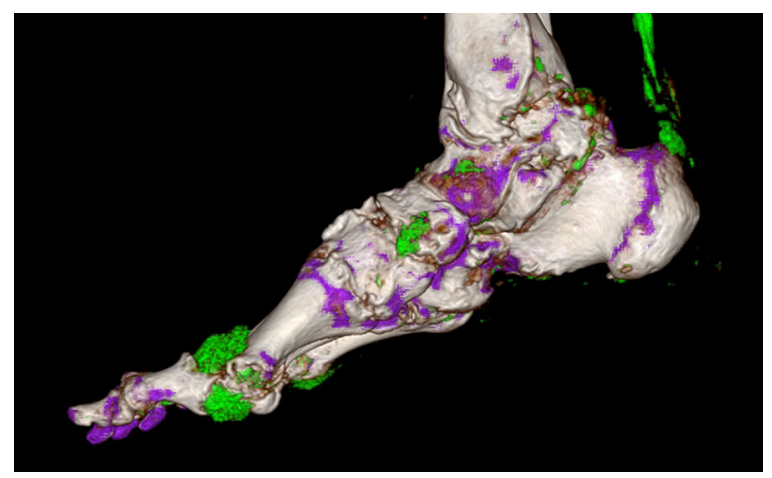

Fig. 2 Three-dimensional volume rendered reconstruction of a dual-energy CT scan of the right foot in a patient with tophaceous gout, demonstrating the extent of urate crystal deposition throughout the foot, and particularly at the 1st metatarsophalangeal joint and Achilles tendon. Urate crystals are shown in green 
many people with hyperuricemia do not develop clinically apparent disease [25]. Therefore, other factors must also contribute to the development of clinically apparent disease. Recent studies have shown that in those with mean serum urate concentrations of $>8 \mathrm{mg} / \mathrm{dL}$, ultrasonographic features of MSU crystal deposition are present in $17-42 \%$ of individuals [26-29]. Microscopy studies have confirmed the presence of MSU crystals in the majority of those with ultrasonographic appearances of crystal deposition [26]. Thus, it seems likely that MSU crystal deposition occurs as a precursor to clinically apparent disease. At present, it is unknown whether the presence of MSU crystals on ultrasonography can predict development of clinically apparent gout in individuals with hyperuricemia. It is also unknown whether interventions to reduce serum urate concentrations in such individuals with hyperuricemia and imaging features of MSU crystal deposition have a role in reducing the risk of developing recurrent flares or joint damage associated with gout.

Gout is characterized by recurrent flares of severe joint inflammation, and most patients are frequently entirely asymptomatic during intercritical periods. Microscopy studies have indicated that MSU crystals are present in previously inflamed joints in people with gout and persistent hyperuricemia [30]. Advanced imaging methods have provided further insight into these intercritical periods, demonstrating that imaging features of MSU crystal deposition are frequently present in people during intercritical periods or at times when joints are not acutely inflamed [31]. These findings reinforce the concept that gout is a chronic disease of MSU crystal deposition and that crystal deposition can progress even in the absence of clinical features of joint inflammation.
The extent of MSU crystal deposition in people with gout has also been highlighted by advanced imaging methods, particularly DECT. This technique demonstrates many more urate deposits than can be appreciated by physical examination [24]. In addition, DECT and ultrasonography have clarified the patterns of crystal deposits, particularly in periarticular sites. A detailed ultrasonography study has identified both cartilage and tendon areas with high frequency (>35\%) of MSU crystal deposition as the 1st metatarsal dorsal cartilage, patellar tendon, 1st metatarsophalangeal dorsal recess, triceps tendon, femoral condyle cartilage, quadriceps tendon and radiocarpal dorsal recess [32]. In contrast, other areas are infrequently affected $(<5 \%) \quad$ including the 1st-5th metacarpophalangeal palmar recess, elbow posterior recess, deep infrapatellar bursa, gastrocnemius-semimembranosus bursa, and 5th metacarpophalangeal dorsal and palmar cartilage [32]. Similarly, a detailed DECT analysis of foot scans in people with tophaceous gout has shown that urate crystal deposition is as frequently located in tendon sites as bone sites, and that certain tendons, particularly the Achilles tendon and peroneal tendons are more frequently affected [33]. Such tendon involvement is often difficult to appreciate on physical examination.

A further insight from imaging studies has been the close relationship between MSU crystal deposition and joint damage. In people with advanced gout, bone erosion is a frequent finding on plain radiography. In addition, other structural damage including joint space narrowing and features of new bone formation such as bone sclerosis and spur formation are frequently observed [34]. Conventional CT and DECT studies have demonstrated the close relationship between features of structural 


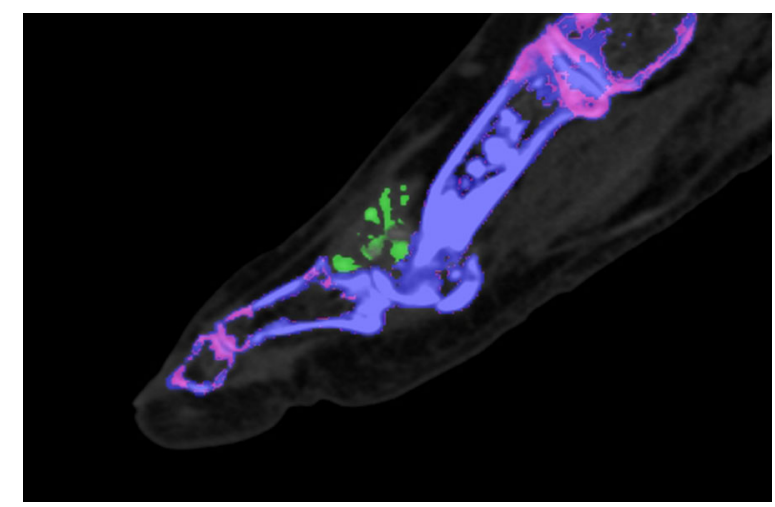

Fig. 3 Two-dimensional DECT sagittal image demonstrating urate crystal deposition within an erosion at the 1st metatarsophalangeal joint in a patient with tophaceous gout. Urate crystals are shown in green

joint damage and urate deposition, with MSU crystals frequently observed within areas of bone erosion [34-36] (Fig. 3). Structural change is a definite indication for uratelowering therapy. However, the recent reports demonstrating a close relationship between joint damage and MSU crystal deposition emphasize the importance of strategies to reduce crystal burden before structural damage occurs.

The role of imaging in clinical management of gout is rapidly changing, as we gain further understanding about the various methods. Both ultrasonography and DECT can play a role in gout diagnosis, although diagnostic accuracy is not perfect; a recent meta-analysis comparing advanced imaging for gout with microscopic diagnosis has reported a pooled sensitivity and specificity of US double contour sign as 0.83 and 0.76 , respectively; of US tophus as 0.65 and 0.80 , respectively; and of DECT as 0.87 and 0.84, respectively [37]. Therefore, although advanced imaging may assist with gout diagnosis, in the situation of clinical uncertainty, microscopic diagnosis remains the gold standard. In clinical practice, imaging can also play an important role in assessment of disease complications, including involvement of tendons/ligaments, spinal disease [38], structural joint damage, and bone and soft tissue infection that can mimic acute gout flares. In the context of possible infection, magnetic resonance imaging (MRI) can play a particularly important role, with the presence of MRI bone marrow edema strongly suggestive of infection rather than gout [39]. Although the costs and ionizing radiation of DECT preclude the widespread use for serial monitoring of disease in most clinical situations, ultrasonography within the clinic may be a particularly useful monitoring tool in a number of clinical scenarios, including in patients who have achieved target serum urate concentrations but have ongoing symptomatic disease, and in those who have incomplete adherence to urate-lowering therapy [40, 41]. Both ultrasonography and DECT can also play an important role in patient understanding of disease both at the time of diagnosis and during follow-up; visualization of crystals using imaging methods allows the patient to understand the central concept of gout as a chronic disease of MSU crystal deposition, and the need for long-term urate-lowering therapy to reduce serum urate and ultimately achieve dissolution of MSU crystals.

\section{TREATING HYPERURICEMIA IN GOUT: THE IMPORTANCE OF GETTING TO TARGET}

Strategies for treatment to target (T2T) have become extremely popular lately in the case of diverse diseases such as in diabetes, hypertension, hyperlipidemia, and rheumatoid arthritis. Targets have also been recommended when facing the treatment of hyperuricemia in gout, depending on actual clinical stage of disease, but also for long-term treatment. 
The strategy to achieve gout "cure" [42] is first to completely dissolve MSU crystals and second, once no crystal burden is present, to avoid new crystal formation [43]. The overall strategy is based on tactical movements or interventions to achieve strategic outcomes.

One of the first tactics to achieve victory when facing patients with gout is to fully understand, as health care providers, the importance and impact of proper information and proactive education of patients [44]. These actions have been nicely shown to improve adherence, avoid withdrawal of treatment and reduce loss to follow-up. Time restriction in clinics may jeopardize such an approach, but investing time for definitive diagnosis, information, and education will surely enhance success [44].

Implementing anti-inflammatory prophylaxis prior to or at the initiation of urate-lowering treatment (ULT), generally for 6-12 months duration, to prevent the occurrence of acute episodes of inflammation (flares) should be seriously considered, and is highly recommended [2, 45], although medications and doses labeled may widely differ among different countries. In addition, patients should be instructed about how to manage the acute episodes of inflammation that may appear despite adequate prophylaxis, mostly in the first year after initiating uratelowering therapy.

Reducing sUA levels to target is the main strategic concept to "cure" gout [46] and is pathophysiologically sound, inverting the mechanism of MSU crystal formation. Different targets have been recognized and these targets may also differ considering the burden of deposition, or stages of the natural progression of the disease [47] and different stages of treatment, initially dissolving pre-formed crystals, then preventing new crystal formation [43].
Treating to target sUA comprises the concept that ULT should reach and maintain steady subsaturating sUA levels in plasma in the long term $[2,3]$, that is sUA at least lower than $6 \mathrm{mg} /$ dl $(360 \mu \mathrm{mol} / \mathrm{L})$ in all patients with gout, as there is compelling evidence that getting to this minimum target is associated with reduction of flares and tissue deposits [48]. Nevertheless, as discussed in the 2006 European League Against Rheumatism (EULAR) recommendations [2], the 2012 American College of Rheumatology (ACR) guidelines recommend that targeting lower that $5 \mathrm{mg} / \mathrm{dl}(300 \mu \mathrm{mol} / \mathrm{L})$ is required for those patients with extensive MSU crystal deposition on either clinical examination or imaging studies [3], as the lower sUA levels achieved, the more rapid reduction of deposits in tissues [40, 49].

Although lifelong control of sUA to subsaturating levels is recommended, the previously cited recommendations and guidelines do not approach whether these targets should be maintained lifelong, that is, after the last MSU crystal has dissolved due to appropriate long-term ULT. On this point, some patients may not need ULMs lifelong as after long-term treatment they may show subsaturating sUA levels [50]. Therefore, a twostage approach to urate lowering can be designed: a first intensive urate-lowering approach to completely dissolve MSU crystals, and especially for those patients with extensive deposition, and afterwards just keep sUA to just below the saturation threshold lifelong. To make it clear: "more is required to get it clean than to keep it clean" [43].

ULMs are to be considered in patients with a definite diagnosis of gout, the several guidelines and recommendations differing in the clinical starting point for prescribing ULMs. Monotherapy with xanthine-oxidase inhibitors has been recommended as first-line treatment, 
medications that exert uricosuric activity to be added or to be considered in monotherapy if the desirable initial target sUA is not achieved [3]. Add-on regimens combining allopurinol and probenecid [51] and allopurinol and benzbromarone [49] have shown to further reduce sUA, a fact that may be especially useful to rapidly dissolve tophi [49]. Phase 1 and 2 clinical trials have also shown the effectiveness in getting to target combining lesinurad with allopurinol [52] and lesinurad with febuxostat [53].

In summary, the prevalence of hyperuricemia and gout is increasing, deposition of MSU crystals and subclinical inflammation being detected in advance of the appearance of the first clinical symptom of gout. The relationship of hyperuricemia, and more clearly of gout, on cardiovascular outcomes has raised increasing interest. The usefulness of imaging techniques to evaluate the presence and the extent of MSU crystal deposition and as outcomes for urate-lowering treatment looks promising, especially ultrasonography and DECT. Such MSU crystal deposits can be effectively and rapidly dissolved with targeted urate-lowering therapy, and neglecting a curable disease should be avoided. The armamentarium to achieve such a goal has increased in recent years and new medications are in the pipeline.

\section{ACKNOWLEDGMENTS}

Open access fee for this article was funded by AstraZeneca. The content of this paper is based on the symposia held in EULAR meeting in Paris in June 2014, sponsored by AstraZeneca. The content is strictly based at the discretion of the authors. AstraZeneca was invited to carry out a courtesy scientific accuracy review prior to peer review for any inaccuracy related to the contents of the meeting, but not influencing the content. The authors have not received any honorary or financial support to write this paper. All named authors meet the ICMJE criteria for authorship for this manuscript, take responsibility for the integrity of the work as a whole, and have given final approval for the version to be published.

All Figures are copyrighted by the authors, with permission to be used in this publication.

Conflict of interest. Fernando Perez-Ruiz has received consulting or speaker fees from AstraZeneca, Menarini, Novartis, Pfizer, and SOBI; investigation grants from Asociación de Reumatólogos del Hospital de Cruces.

Nicola Dalbeth has received consulting fees, speaker fees or grants from the following companies: Takeda, Teijin, Menarini, Ardea, AstraZeneca, Savient, Fonterra, Metabolex.

Tomas Bardin has received consulting fees, speaker fees or grants from the following companies: Ardea, Asstellas, AstraZeneca, Ipsen, Menarini, Novartis, Savient, Sobi, Takeda.

Open Access. This article is distributed under the terms of the Creative Commons Attribution Noncommercial License which permits any noncommercial use, distribution, and reproduction in any medium, provided the original author(s) and the source are credited.

\section{REFERENCES}

1. Bardin T, Richette P. Definition of hyperuricemia and gouty conditions. Curr Opin Rheumatol. 2014;26:186-91.

2. Zhang W, Doherty M, Pascual E, Bardin T, Barskova V, Conagham P, Gerster J, et al. EULAR evidence based recommendations for gout Part II. Management. Report of a Task Force of the EULAR 
Standing Committee for international clinical studies including therapeutics (ESCISIT). Ann Rheum Dis. 2006;65:1312-24.

3. Khanna D, Fitzgerald JD, Khanna PP, Bae S, Singh MK, Neogi T, et al. 2012 American College of Rheumatology guidelines for management of gout. Part 1: systematic nonpharmacologic and pharmacologic therapeutic approaches to hyperuricemia. Arthritis Care Res (Hoboken). 2012;64:1431-46.

4. Richette P, Perez-Ruiz F, Doherty M, Jansen TL, Nuki G, Pascual E, et al. Improving cardiovascular and renal outcomes in gout: what should we target? Nat Rev Rheumatol. 2014;. doi:10.1038/nrrheum. 2014.124 .

5. Smith EU, Diaz-Torne C, Perez-Ruiz F, March LM. Epidemiology of gout: an update. Best Pract Res Clin Rheumatol. 2010;24:811-27.

6. Perez-Ruiz F, Martinez-Indart L, Carmona L, Herrero-Beites AM, Pijoan JI, Krishnan E. Tophaceous gout and high level of hyperuricaemia are both associated with increased risk of mortality in patients with gout. Ann Rheum Dis. 2014;73:177-82.

7. Choi HK, Curhan G. Independent impact of gout on mortality and risk for coronary heart disease. Circulation. 2007;116:894-900.

8. Krishnan E, Svendsen K, Neaton JD, Grandits G, Kuller LH. Long-term cardiovascular mortality among middle-aged men with gout. Arch Intern Med. 2008;168:1104-10.

9. Stack AG, Hanley A, Casserly LF, Cronin CJ, Abdalla $\mathrm{AA}$, Kiernan TJ, et al. Independent and conjoint associations of gout and hyperuricemia with total and cardiovascular mortality. QJM. 2013;106:647-58.

10. Culleton BF, Larson MG, Kannel WB, Levy D. Serum uric acid and risk for cardiovascular disease and death: the Framingham Heart Study. Ann Intern Med. 1999;131:7-13.

11. Zhu Y, Pandya BJ, Choi HK. Comorbidities of gout and hyperuricemia in the US general population: NHANES 2007-2008. Am J Med. 2012;125:679-87.

12. Grayson PC, Kim SY, LaValley M, Choi HK. Hyperuricemia and incident hypertension: a systematic review and meta-analysis. Arthritis Care Res (Hoboken). 2011;63:102-10.

13. Johnson RJ, Kang DH, Feig DI, Kivlighn S, Kanellis J, Watanabe $S$, et al. Is there a pathogenetic role for uric acid in hypertension and cardiovascular and renal disease? Hypertension. 2003;41:1183-90.
14. Sanchez-Lozada LG, Tapia E, Bautista-Garcia P, Soto V, Vila-Casado C, Vega-Campos IP, et al. Effects of febuxostat on metabolic and renal alterations in rats with fructose-induced metabolic syndrome. Am J Physiol Renal Physiol. 2008;294:F710-8.

15. DeBosch BJ, Kluth O, Schürmann A, Moley K. Early-onset metabolic syndrome in mice lacking the intestinal uric acid transporter SLC2A9. Nat Commun. 2014;5:4642. doi:10.1038/ncomms 5642 .

16. Feig DI, Soletsky B, Johnson RJ. Effect of allopurinol on blood pressure of adolescents with newly diagnosed essential hypertension. A randomized trial. JAMA. 2008;300:925-32.

17. Soletsky B, Feig DI. Uric acid reduction rectifies prehypertension in obese adolecents. Hypertension. 2012;60:1148-56.

18. Kawashima M, Wada $K$, Ohta $H$, Terawaki $H$, Aizawa Y. Association between asymptomatic hyperuricemia and new-onset chronic kidney disease in Japanese male workers: a long-term retrospective cohort study. BMC Nephrol. 2011;12:31. doi:10.1186/1471-2369-12-31.

19. Grimaldi-Bensouda L, Alperovitch A, Aubrun E, Danchin N, Rossignol M, Abenhaim L, et al. Impact of allopurinol on risk of myocardial infarction. Ann Rheum Dis. 2012. doi:10.1136/annrheumdis-2012202972 (Epub ahead of print).

20. Wei L, Mackenzie IS, Chen Y, Struthers AD, MacDonald TM. Impact of allopurinol use on urate concentration and cardiovascular outcome. Br J Clin Pharmacol. 2011;71:600-7.

21. Grassi W, Meenagh G, Pascual E, Filippucci E. "Crystal Clear"-sonographic assessment of gout and calcium pyrophosphate deposition disease. Semin Arthritis Rheum. 2006;36:197-202.

22. Thiele RG, Schlesinger $N$. Ultrasonography is a reliable, non-invasive method for diagnosing gout. Rheumatology. 2007;46:1116-21.

23. Palmer DG, Highton J, Hessian PA. Development of the gout tophus. An hypothesis. Am J Clin Pathol. 1989;91:190-5.

24. Choi HK, Al-Arfaj A, Eftekhari A, Munk PL, Shojania $\mathrm{K}$, Reid G, et al. Dual energy computed tomography in tophaceous gout. Ann Rheum Dis. 2009;. doi:10. 1136/ard.2008.099713.

25. Campion EW, Glynn RJ, DeLabry LO. Asymptomatic hyperuricemia. Risks and consequences in the normative aging study. Am J Med. 1987;82:421-6. 
26. De Miguel E, Puig JG, CastilloC, Peiteado D, Torres RJ, Martin-Mola E. Diagnosis of gout in patients with asymptomatic hyperuricaemia: a pilot ultrasound study. Ann Rheum Dis. 2011;71:157-8.

27. Howard RG, Pillinger MH, Gyftopoulos S, Thiele RG, Swearingen CJ, Samuels J. Reproducibility of musculoskeletal ultrasound for determining monosodium urate deposition: concordance between readers. Arthritis Care Res (Hoboken). 2011;. doi:10.1002/acr.20527.

28. Pineda C, Amezcua-Guerra LM, Solano C, Rodriguez-Henriquez P, Hernandez-Diaz C, Vargas $A$, et al. Joint and tendon subclinical involvement suggestive of gouty arthritis in asymptomatic hyperuricemia: an ultrasound controlled study. Arthritis Res Ther. 2011;13:R4.

29. Puig JG, de Miguel E, Castillo MC, Rocha AL, Martinez MA, Torres RJ. Asymptomatic hyperuricemia: impact of ultrasonography. Nucleosides Nucleotides Nucleic Acids. 2008;27:592-5.

30. Pascual E, Batlle-Gualda E, Martinez A, Rosas J, Vela P. Synovial fluid analysis for diagnosis of intercritical gout. Ann Intern Med. 1999;131:756-9.

31. Schauer C, Janko C, Munoz LE, Zhao Y, Kienhöfer $D$, et al. Aggregated neutrophil extracellular traps limit imflammation by degrading cytokines and chemokines. Nat Med. 2014;20:511-7.

32. Naredo E, Uson J, Jimenez-Palop M, Martinez A, Vicente E, Brito E, et al. Ultrasound-detected musculoskeletal urate crystal deposition: which joints and what findings should be assessed for diagnosing gout? Ann Rheum Dis. 2013; doi:10. 1136/annrheumdis-2013-203487 (Epub ahead of print).

33. Dalbeth N, Kalluru R, Aati O, Horne A, Doyle AJ, McQueen FM. Tendon involvement in the feet of patients with gout: a dual-energy CT study. Ann Rheum Dis. 2013;72:1545-8.

34. Dalbeth N, Milligan A, Doyle AJ, Clark B, McQueen FM. Characterization of new bone formation in gout: a quantitative site-by-site analysis using plain radiography and computed tomography. Arthritis Res Ther. 2012;14:R165.

35. Dalbeth N, Clark B, Gregory K, Gamble G, Sheehan $\mathrm{T}$, Doyle A, et al. Mechanisms of bone erosions in gout: a quantitative analysis using plain radiography and computed tomography. Ann Rheum Dis. 2008;. doi:10.1136/ard.2008.094201.

36. Dalbeth N, Aati O, Kalluru R, Gamble GD, Horne A, Doyle AJ, et al. Relationship between structural joint damage and urate deposition in gout: a plain radiography and dual-energy CT study. Ann Rheum Dis. 2014; . doi:10.1136/annrheumdis-2013-204273 (Epub ahead of print).

37. Ogdie A, Taylor WJ, Weatherall M, Fransen J, Jansen TL, Neogi T, et al. Imaging modalities for the classification of gout: systematic literature review and meta-analysis. Ann Rheum Dis. 2014; doi:10.1136/annrheumdis-2014-205431 (Epub ahead of print).

38. Konatalapalli RM, Lumezanu E, Jelinek JS, Murphey MD, Wang H, Weinstein A. Correlates of axial gout: a cross-sectional study. J Rheumatol. 2012;39:1445-9.

39. Poh YJ, Dalbeth N, Doyle A, McQueen FM. Magnetic resonance imaging bone edema is not a major feature of gout unless there is concomitant osteomyelitis: 10-year findings from a highprevalence population. J Rheumatol. 2011;38: 2475-81.

40. Perez-Ruiz F, Martin I, Canteli B. Ultrasonographic measurement of tophi as an outcome measure for chronic gout. J Rheumatol. 2007;34:1888-93.

41. Thiele RG, Schlesinger N. Ultrasonography shows disappearance of monosodium urate crystal deposition on hyaline cartilage after sustained normouricemia is achieved. Rheumatol Int. 2009; doi:10.1007/s00296-009-1002-8.

42. Doherty M, Jansen TL, Nuki G, Pascual E, Perez-Ruiz F, Punzi L, et al. Gout: why is this curable disease so seldom cured? Ann Rheum Dis. 2012;71:1765-70.

43. Perez-Ruiz F, Herrero-Beites AM, Carmona L. A twostage approach to the treatment of hyperuricemia in gout: the "Dirty Dish" hypothesis. Arthritis Rheum. 2011;63:4002-6.

44. Rees F, Jenkins W, Doherty M. Patients with gout adhere to curative treatment if informed appropriately: proof-of-concept observational study. Ann Rheum Dis. 2013;72:826-30.

45. Khanna D, Khanna PP, Fitzgerald JD, Singh MK, Bae S, Neogi T, et al. 2012 American College of Rheumatology guidelines for management of gout. Part 2: Therapy and antiinflammatory prophylaxis of acute gouty arthritis. Arthritis Care Res (Hoboken). 2012;64:1447-61.

46. Perez-Ruiz F. Treating to target: an strategy to cure gout. Rheumatology. 2009;49:ii9-12.

47. Perez-Ruiz F, Castillo E, Chinchilla SP, HerreroBeites AM. Clinical manifestations and diagnosis of gout. Rheum Dis Clin North Am. 2014;40:193-206.

48. Perez-Ruiz F, Lioté F. Lowering serum uric acid levels: what is the optimal target for improving 
clinical outcomes in gout? Arthritis Rheum. 2007;57:1324-8.

49. Perez-Ruiz F, Calabozo M, Pijoan JI, Herrero-Beites AM, Ruibal A. Effect of urate-lowering therapy on the velocity of size reduction of tophi in chronic gout. Arthritis Rheum. 2002;47:356-60.

50. Perez-Ruiz F, Atxotegi J, Hernando I, Calabozo M, Nolla JM. Using serum urate levels to determine the period free of gouty symptoms after withdrawal of long-term urate-lowering therapy: a prospective study. Arthritis Rheum. 2006;55:786-90.

51. Reinders MK, Van Roon EN, Houtman PM, Brouwers JR, Jansen TL. Biochemical effectiveness of allopurinol and allopurinol-probenecid in previously benzbromarone-treated gout patients. Clin Rheumatol. 2007;26:1459-65.
52. Perez Ruiz F, Sundy J, Krishnan E, Hingorani V, Welp J, Rodgers T, et al. Efficacy and safety of lesinurad (RDEA594), a novel URAT1 inhibitor, in combination with allopurinol-refractory gout patients: results from a randomized, blinded, placebo controlled, phase $2 \mathrm{~B}$ extension study. Ann Rheum Dis. 2012;71:439.

53. Fleischmann R, Kerr B, Yeh LT, Suster M, Shen Z, Polvent E, et al. Pharmacodynamic, pharmacokinetic and tolerability evaluation of concomitant administration of lesinurad and febuxostat in gout patients with hyperuricaemia. Rheumatology (Oxford). 2014;. doi:10.1093/ rheumatology $/$ ket 487 . 